\title{
Some identities of degenerate Daehee numbers arising from certain differential equations
}

\author{
Taekyun Kimª,b,*, Dae San Kim \\ ${ }^{a}$ Department of Mathematics, College of Science Tianjin Polytechnic University, Tianjin 300160, China. \\ ${ }^{b}$ Department of Mathematics, Kwangwoon University, Seoul 139-701, Republic of Korea. \\ ${ }^{c}$ Department of Mathematics, Sogang University, Seoul 121-742, Republic of Korea.
}

Communicated by W. Shatanawi

\begin{abstract}
In this paper, we introduce the degenerate Daehee numbers and study a family of differential equations associated with the generating function of these numbers. From those differential equations, we will be able to obtain some new and interesting combinatorial identities involving the degenerate Daehee numbers and generalized harmonic numbers. (C)2017 All rights reserved.
\end{abstract}

Keywords: Degenerate Daehee numbers, differential equation, generalized harmonic numbers. 2010 MSC: 05A19, 11B83, 34A34.

\section{Introduction}

The Daehee polynomials $D_{n}^{(r)}(x)$ of order $r$ are given by the generating function

$$
\left(\frac{\log (1+t)}{t}\right)^{r}(1+t)^{r}=\sum_{n=0}^{\infty} D_{n}^{(r)}(x) \frac{t^{n}}{n !} .
$$

For $x=0, D_{n}^{(r)}=D_{n}^{(r)}(0)$ are called the Daehee numbers of order $r$. In particular, if $r=1$, then $D_{n}(x)=D_{n}^{(1)}(x)$ and $D_{n}=D_{n}^{(1)}$ are respectively called Daehee polynomials and Daehee numbers (see $[1,6,8,10,12,18-22,24])$.

As a degenerate version of Daehee numbers $D_{n}$, we introduce what we call the degenerate Daehee numbers $D_{n, \lambda}$ defined by

$$
\frac{\lambda \log \left(1+\frac{1}{\lambda} \log (1+\lambda t)\right)}{\log (1+\lambda t)}=\sum_{n=0}^{\infty} D_{n, \lambda} \frac{t^{n}}{n !} .
$$

\footnotetext{
*Corresponding author

Email addresses: tkkim@kw.ac.kr (Taekyun Kim), dskim@sogang.ac.kr (Dae San Kim)
} doi:10.22436/jnsa.010.02.35 
We observe here that $D_{n, \lambda} \rightarrow D_{n}$ as $\lambda \rightarrow 0$. Also it is easy to see that

$$
D_{n, \lambda}=\sum_{l=0}^{n} S_{l}(n, l) \lambda^{n-l} D_{l}=\sum_{l=0}^{n} \frac{(-1)^{l} l !}{l+1} S_{1}(n, l) \lambda^{n-l} .
$$

Here $S_{1}(n, l)$ is the Stirling number of the first kind.

Many mathematicians have studied the arithmetic and combinatorial properties of degenerate versions of special numbers and polynomials, some of which are the degenerate Bernoulli polynomials (also called Korobov polynomials of the second kind), the degenerate Bernouili polynomials of the second kind (also called Korobov polynomials of the first kind), the degenerate Euler polynomials, the degenerate polyBernoulli polynomials, the degenerate poly-Bernoulli polynomials of the second, the degenerate falling factorial polynomials, and the degenerate Changhee polynomials (see $[2-5,9,12,14,16,17,23])$.

On the other hand, in [7, 11], Kim et al. developed some new methods for obtaining identities related to Bernoulli numbers of the second kind and Frobenius-Euler polynomials of higher order arising from certain non-linear differential equations. This idea of obtaining some interesting combinatorial identities by using differential equations satisfied by the generating function of special numbers or special polynomials turned out to be very fruitful (see [7, 11, 13, 15]).

The generalized harmonic numbers are defined as follows:

$$
\begin{aligned}
& \mathrm{H}_{\mathrm{N}, 0}=1, \quad \text { for all } \mathrm{N}, \\
& \mathrm{H}_{\mathrm{N}, 1}=\mathrm{H}_{\mathrm{N}}=\frac{1}{N}+\frac{1}{\mathrm{~N}-1}+\cdots+\frac{1}{1^{\prime}} \\
& \mathrm{H}_{\mathrm{N}, \mathrm{j}}=\frac{\mathrm{H}_{\mathrm{N}-1, j-1}}{\mathrm{~N}}+\frac{\mathrm{H}_{\mathrm{N}-2, j-1}}{\mathrm{~N}-1}+\cdots+\frac{\mathrm{H}_{j-1, j-1}}{j}, \quad(2 \leqslant j \leqslant N) .
\end{aligned}
$$

These special numbers have appeared previously in the paper [11].

The purpose of this paper is to introduce the degenerate Daehee numbers and study a family of differential equations associated with the generating function of these numbers. From those differential equations, we will be able to obtain some new and interesting combinatorial identities involving the degenerate Daehee numbers and generalized harmonic numbers.

\section{Differential equations arising from the generating function of degenerate Daehee numbers}

Let

$$
\mathrm{F}(\mathrm{t})=\mathrm{F}=\log \left(1+\frac{1}{\lambda} \log (1+\lambda \mathrm{t})\right) .
$$

Then by taking the derivative with respect to $t$ of (2.1), we get

$$
\begin{aligned}
F^{(1)} & =\frac{d}{d t} F(t)=\left(1+\frac{1}{\lambda} \log (1+\lambda t)\right)^{-1} \frac{1}{1+\lambda t} \\
& =\frac{1}{1+\lambda t} e^{-\log \left(1+\frac{1}{\lambda} \log (1+\lambda t)\right)} \\
& =\frac{1}{1+\lambda} e^{-F} .
\end{aligned}
$$

From (2.2), we note that

$$
\begin{aligned}
F^{(2)} & =\frac{d}{d t} F^{(1)}=\frac{(-1) \lambda}{(1+\lambda t)^{2}} e^{-F}+\frac{1}{1+\lambda t}\left(-F^{(1)}\right) e^{-F} \\
& =\frac{(-1) \lambda}{(1+\lambda t)^{2}} e^{-F}+\frac{(-1)}{(1+\lambda t)^{2}} e^{-2 F} .
\end{aligned}
$$


Further, by taking the derivative with respect to $t$ of (2.3), we obtain

$$
\begin{aligned}
F^{(3)}= & \frac{d}{d t} F^{(2)} \\
= & \frac{(-1)^{2} 2 \lambda^{2}}{(1+\lambda t)^{3}} e^{-F}+\frac{(-1) \lambda}{(1+\lambda t)^{2}}\left(-F^{(1)}\right) e^{-F} \\
& +\frac{(-1)^{2} 2 \lambda}{(1+\lambda t)^{3}} e^{-2 F}+\frac{(-1)}{(1+\lambda t)^{2}}\left(-2 F^{(1)}\right) e^{-2 F} \\
= & \frac{(-1)^{2}}{(1+\lambda t)^{3}}\left(2 \lambda^{2} e^{-F}+3 \lambda e^{-2 F}+2 e^{-3 F}\right) .
\end{aligned}
$$

Continuing this process, we are led to put

$$
F^{(N)}=\frac{(-1)^{N-1}}{(1+\lambda t)^{N}} \sum_{k=1}^{N} a_{k}(N \mid \lambda) e^{-k F},
$$

for $\mathrm{N}=1,2,3, \cdots$.

On the one hand, from (2.4) we have

$$
\begin{aligned}
F^{(N+1)=} & \frac{d}{d t} F^{(N)} \\
= & \frac{(-1)^{N} N \lambda}{(1+\lambda t)^{N+1}} \sum_{k=1}^{N} a_{k}(N \mid \lambda) e^{-k F} \\
& +\frac{(-1)^{N-1}}{(1+\lambda t)^{N}} \sum_{k=1}^{N} a_{k}(N \mid \lambda)\left(-k F^{(1)}\right) e^{-k F} \\
= & \frac{(-1)^{N} N \lambda}{(1+\lambda t)^{N+1}} \sum_{k=1}^{N} a_{k}(N \mid \lambda) e^{-k F} \\
& +\frac{(-1)^{N}}{(1+\lambda t)^{N+1}} \sum_{k=1}^{N} a_{k}(N \mid \lambda) k e^{-(k+1) F} \\
= & \frac{(-1)^{N}}{(1+\lambda t)^{N+1}} \sum_{k=1}^{N} \lambda N a_{k}(N \mid \lambda) e^{-k F} \\
& +\frac{(-1)^{N}}{(1+\lambda t)^{N+1}} \sum_{k=2}^{N+1}(k-1) a_{k-1}(N \mid \lambda) e^{-k F} \\
= & \frac{(-1)^{N}}{(1+\lambda t)^{N+1}}\left\{\lambda N a_{1}(N \mid \lambda) e^{-F}+\sum_{k=2}^{N}\left(\lambda N a_{k}(N \mid \lambda)+(k-1) a_{k-1}(N \mid \lambda)\right) e^{-k F}\right. \\
& \left.+N a_{N}(N \mid \lambda) e^{-(N+1) F}\right\} .
\end{aligned}
$$

On the other hand, by replacing $\mathrm{N}$ by $\mathrm{N}+1$ in (2.4), we get

$$
F^{(N+1)}=\frac{(-1)^{N}}{(1+\lambda t)^{N+1}} \sum_{k=1}^{N+1} a_{k}(N+1 \mid \lambda) e^{-k F} .
$$

Now, by comparing (2.5) and (2.6), we have

$$
a_{1}(N+1 \mid \lambda)=\lambda N a_{1}(N \mid \lambda),
$$




$$
\begin{aligned}
a_{N+1}(N+1 \mid \lambda) & =N a_{N}(N \mid \lambda), \\
a_{k}(N+1 \mid \lambda) & =\lambda N a_{k}(N \mid \lambda)+(k-1) a_{k-1}(N \mid \lambda),
\end{aligned}
$$

where $2 \leqslant k \leqslant N$.

From (2.2) and (2.4), we note

$$
F^{(1)}=\frac{1}{1+\lambda t} e^{-F}=a_{1}(1 \mid \lambda) \frac{1}{1+\lambda t} e^{-F}
$$

Thus by (2.10), we obtain

$$
a_{1}(1 \mid \lambda)=1 .
$$

In addition, from (2.3) and (2.4), we observe

$$
\begin{aligned}
F^{(2)} & =\frac{(-1) \lambda}{(1+\lambda t)^{2}} e^{-F}+\frac{(-1)}{(1+\lambda t)^{2}} e^{-2 F} \\
& =\frac{(-1)}{(1+\lambda t)^{2}}\left(a_{1}(2 \mid \lambda) e^{-F}+a_{2}(2 \mid \lambda) e^{-2 F}\right) .
\end{aligned}
$$

Hence, from (2.11) we have

$$
\mathrm{a}_{1}(2 \mid \lambda)=\lambda, \quad \mathrm{a}_{2}(2 \mid \lambda)=1 .
$$

Now, we are ready to determine $a_{k}(N+1 \mid \lambda)$ 's appearing in (2.7), (2.8) and (2.9). From (2.7), we get

$$
\begin{aligned}
a_{1}(N+1 \mid \lambda) & =\lambda N a_{1}(N \mid \lambda) \\
& =\lambda N \lambda(N-1) a_{1}(N-1 \mid \lambda) \\
& \vdots \\
& =(\lambda N) \lambda(N-1) \cdots \lambda 2 a_{1}(2 \mid \lambda) \\
& =\lambda^{N} N ! .
\end{aligned}
$$

By (2.8), we have

$$
\begin{aligned}
a_{N+1}(N+1 \mid \lambda) & =N a_{N}(N \mid \lambda) \\
& =N(N-1) a_{N-1}(N-1 \mid \lambda) \\
& \vdots \\
& =N(N-1) \cdots 2 a_{2}(2 \mid \lambda) \\
& =N ! .
\end{aligned}
$$
by

We remark here that the $N \times N$ matrix with the $(i, j)$ entry given by $a_{i}(j \mid \lambda)(1 \leqslant i, j \leqslant n)$ is defined

$$
\left[\begin{array}{ccccc}
1 & \lambda & \lambda^{2} 2 ! & \cdots & \lambda^{\mathrm{N}-1}(\mathrm{~N}-1) ! \\
0 & 2 ! & & & \\
0 & 0 & 3 ! & & \\
\vdots & \vdots & \ddots & \ddots & \\
0 & 0 & \cdots & 0 & (\mathrm{~N}-1) !
\end{array}\right]
$$

We now turn our attention to $a_{k}(N+1 \mid \lambda)$, for $2 \leqslant k \leqslant N$. For $k=2$ in (2.9), we have

$$
\begin{aligned}
\mathrm{a}_{2}(\mathrm{~N}+1 \mid \lambda) & =\lambda \mathrm{Na}_{2}(\mathrm{~N} \mid \lambda)+\mathrm{a}_{1}(\mathrm{~N} \mid \lambda) \\
& =\lambda \mathrm{Na}_{2}(\mathrm{~N} \mid \lambda)+\lambda^{\mathrm{N}-1}(\mathrm{~N}-1) !
\end{aligned}
$$




$$
\begin{aligned}
& =\lambda N\left(\lambda(N-1) a_{2}(N-1 \mid \lambda)+\lambda^{N-2}(N-2) !\right)+\lambda^{N-1}(N-1) ! \\
& =\lambda^{2} N(N-1) a_{2}(N-1 \mid \lambda)+\lambda^{N-1} N !\left(\frac{1}{N-1}+\frac{1}{N}\right) \\
& =\lambda^{2} N(N-1)\left(\lambda(N-2) a_{2}(N-2 \mid \lambda)+\lambda^{N-3}(N-3) !\right)+\lambda^{N-1} N !\left(\frac{1}{N-1}+\frac{1}{N}\right) \\
& =\lambda^{3} N(N-1)(N-2) a_{2}(N-2 \mid \lambda)+\lambda^{N-1} N !\left(\frac{1}{N-2}+\frac{1}{N-1}+\frac{1}{N}\right) \\
& \vdots \\
& =\lambda^{N-1} N(N-1) \cdots 2 a_{2}(2 \mid \lambda)+\lambda^{N-1} N !\left(\frac{1}{2}+\frac{1}{3}+\cdots+\frac{1}{N}\right) \\
& =\lambda^{N-1} N ! H_{N, 1} .
\end{aligned}
$$

Here and in below $\mathrm{H}_{\mathrm{N}, \mathrm{j}}(0 \leqslant j \leqslant \mathrm{~N})$ are as defined in (1.2), (1.3) and (1.4).

For $k=3$ in (2.9), we obtain

$$
\begin{aligned}
a_{3}(N+1 \mid \lambda)= & \lambda N a_{3}(N \mid \lambda)+2 a_{2}(N \mid \lambda) \\
= & \lambda N a_{3}(N \mid \lambda)+2 ! \lambda^{N-2}(N-1) ! H_{N-1,1} \\
= & \lambda N\left(\lambda(N-1) a_{3}(N-1 \mid \lambda)+2 ! \lambda^{N-3}(N-2) ! H_{N-2,1}\right) \\
& +2 ! \lambda^{N-2}(N-1) ! H_{N-1,1} \\
= & \lambda^{2} N(N-1) a_{3}(N-1 \mid \lambda)+2 ! \lambda^{N-2} N !\left(\frac{H_{N-2,1}}{N-1}+\frac{H_{N-1,1}}{N}\right) \\
= & \lambda^{2} N(N-1)\left(\lambda(N-2) a_{3}(N-2 \mid \lambda)+2 ! \lambda^{N-4}(N-3) ! H_{N-3,1}\right) \\
& +2 ! \lambda^{N-2} N !\left(\frac{H_{N-2,1}}{N-1}+\frac{H_{N-1,1}}{N}\right) \\
= & \lambda^{3} N(N-1)(N-2) a_{3}(N-2 \mid \lambda)+2 ! \lambda^{N-2} N !\left(\frac{H_{N-3,1}}{N-2}+\frac{H_{N-2,1}}{N-1}+\frac{H_{N-1,1}}{N}\right) \\
\vdots & \\
= & \lambda^{N-2} N(N-1) \cdots 3 a_{3}(3 \mid \lambda)+2 ! \lambda^{N-2} N !\left(\frac{H_{2,1}}{3}+\frac{H_{3,1}}{4}+\cdots+\frac{H_{N-1,1}}{N}\right) \\
= & 2 ! \lambda^{N-2} N !\left(\frac{H_{1,1}}{2}+\frac{H_{2,1}}{3}+\cdots+\frac{H_{N-1,1}}{N}\right) \\
= & 2 ! \lambda^{N-2} N ! H_{N, 2} .
\end{aligned}
$$

Proceeding similarly to $k=2$ and $k=3$ cases, we can show that

$$
\mathrm{a}_{4}(\mathrm{~N}+1 \mid \lambda)=3 ! \lambda^{\mathrm{N}-3} \mathrm{~N} ! \mathrm{H}_{\mathrm{N}, 3} \text {. }
$$

Continuing in this fashion, we can find that

$$
a_{k}(N+1 \mid \lambda)=(k-1) ! \lambda^{N-k+1} N ! H_{N, k-1}, \quad(2 \leqslant k \leqslant N) .
$$

Here we observe that (2.14) holds also for $k=1$ and $k=N+1$ (cf. (2.12), (2.13)).

Thus, from (2.14) we obtain the following theorem.

Theorem 2.1. For $N=1,2,3, \cdots$, let us consider the following family of differential equations:

$$
F^{(N)}=\frac{(-1)^{N-1}}{(1+\lambda t)^{N}} \sum_{k=1}^{N}(k-1) ! \lambda^{N-k}(N-1) ! H_{N-1, k-1} e^{-k F},
$$


where

$$
\begin{aligned}
& \mathrm{H}_{\mathrm{N}, 0}=1, \quad \text { for all } \mathrm{N}, \\
& \mathrm{H}_{\mathrm{N}, 1}=\mathrm{H}_{\mathrm{N}}=\frac{1}{\mathrm{~N}}+\frac{1}{\mathrm{~N}-1}+\cdots+\frac{1}{1}, \\
& \mathrm{H}_{\mathrm{N}, \mathrm{j}}=\frac{\mathrm{H}_{\mathrm{N}-1, j-1}}{\mathrm{~N}}+\frac{\mathrm{H}_{\mathrm{N}-2, j-1}}{\mathrm{~N}-1}+\cdots+\frac{\mathrm{H}_{j-1, j-1}}{\mathrm{j}}, \quad(2 \leqslant j \leqslant \mathrm{~N}) .
\end{aligned}
$$

Then the above family of differential equations in (2.15) have a solution

$$
\mathrm{F}=\mathrm{F}(\mathrm{t})=\log \left(1+\frac{1}{\lambda} \log (1+\lambda \mathrm{t})\right)
$$

\section{Applications of differential equations}

Here we will use Theorem 2.1 in order to derive some new and interesting identity involving the degenerate Daehee numbers and generalized harmonic numbers.

From (2.1), we get

$$
\begin{aligned}
F(t) & =\frac{\lambda \log \left(1+\frac{1}{\lambda} \log (1+\lambda t)\right)}{\log (1+\lambda t)} \frac{1}{\lambda} \log (1+\lambda t) \\
& =\left(\sum_{l=0}^{\infty} D_{l, \lambda} \frac{t^{l}}{l !}\right)\left(\sum_{m=1}^{\infty} \frac{(-1)^{m-1}}{m} \lambda^{m-1} t^{m}\right) \\
& =\sum_{n=1}^{\infty}\left(\sum_{l=0}^{n-1} \frac{D_{l, \lambda}}{l !} \frac{(-\lambda)^{n-l-1}}{n-l}\right) t^{n} .
\end{aligned}
$$

On the one hand, from (3.1) we obtain

$$
\begin{aligned}
F^{(N)} & =\left(\frac{d}{d t}\right)^{N} F(t) \\
& =\sum_{n=N}^{\infty}(n)_{N}\left(\sum_{l=0}^{n-1} \frac{D_{l, \lambda}}{l !} \frac{(-\lambda)^{n-l-1}}{n-l}\right) t^{n-N} \\
& =\sum_{n=0}^{\infty}(n+N)_{N}\left(\sum_{l=0}^{n+N-1} \frac{D_{l, \lambda}}{l !} \frac{(-\lambda)^{n+N-l-1}}{n+N-l}\right) t^{n} \\
& =\sum_{n=0}^{\infty}(n+N) !\left(\sum_{l=0}^{n+N-1} \frac{D_{l, \lambda}}{l !} \frac{(-\lambda)^{n+N-l-1}}{n+N-l}\right) \frac{t^{n}}{n !},
\end{aligned}
$$

where $(x)_{N}=x(x-1) \cdots(x-N+1)$, for $N \geqslant 1$ and $(x)_{0}=1$.

Now, we observe that

$$
\begin{aligned}
e^{-k F} & =\sum_{m_{1}=0}^{\infty} \frac{(-k)^{m_{1}}}{m_{1} !} F^{m_{1}} \\
& =\sum_{m_{1}=0}^{\infty}(-k)^{m_{1}} \frac{1}{m_{1} !}\left(\log \left(1+\frac{1}{\lambda} \log (1+\lambda t)\right)\right)^{m_{1}} \\
& =\sum_{m_{1}=0}^{\infty}(-k)^{m_{1}} \sum_{m_{2}=m_{1}}^{\infty} S_{1}\left(m_{2}, m_{1}\right)\left(\frac{1}{\lambda}\right)^{m_{2}} \frac{(\log (1+\lambda t))^{m_{2}}}{m_{2} !}
\end{aligned}
$$




$$
\begin{aligned}
= & \sum_{m_{2}=0}^{\infty}\left(\sum_{m_{1}=0}^{m_{2}}(-k)^{m_{1}} S_{1}\left(m_{2}, m_{1}\right) \lambda^{-m_{2}}\right) \\
& \times \sum_{m_{3}=m_{2}}^{\infty} S_{1}\left(m_{3}, m_{2}\right) \lambda^{m_{3}} \frac{t^{m_{3}}}{m_{3} !} \\
= & \sum_{m_{3}=0}^{\infty}\left(\sum_{m_{2}=0}^{m_{3}} \sum_{m_{1}=0}^{m_{2}}(-k)^{m_{1}} S_{1}\left(m_{2}, m_{1}\right) S_{1}\left(m_{3}, m_{2}\right) \lambda^{m_{3}-m_{2}}\right) \frac{t^{m_{3}}}{m_{3} !} .
\end{aligned}
$$

In turn, (3.3) gives us

$$
\begin{aligned}
\frac{1}{(1+\lambda t)^{N}} e^{-k F}= & \left(\sum_{l=0}^{\infty}\left(\begin{array}{c}
N+l-1 \\
l
\end{array}\right)(-1)^{l} \lambda^{l} t^{l}\right) \\
& \times \sum_{m_{3}=0}^{\infty}\left(\sum_{m_{2}=0}^{m_{3}} \sum_{m_{1}=0}^{m_{2}}(-k)^{m_{1}} S_{1}\left(m_{2}, m_{1}\right) S_{1}\left(m_{3}, m_{2}\right) \lambda^{m_{3}-m_{2}}\right) \frac{t^{m_{3}}}{m_{3} !} \\
= & \sum_{n=0}^{\infty}\left(\sum_{m_{3}=0}^{n} \sum_{m_{2}=0}^{m_{3}} \sum_{m_{1}=0}^{m_{2}}(-1)^{n+m_{1}+m_{3}}\left(\begin{array}{c}
n \\
m_{3}
\end{array}\right)\left(N+n-m_{3}-1\right)_{n-m_{3}}\right. \\
& \left.\times k^{m_{1}} \lambda^{n-m_{2}} S_{1}\left(m_{2}, m_{1}\right) S_{1}\left(m_{3}, m_{2}\right)\right) \frac{t^{n}}{n !} .
\end{aligned}
$$

On the other hand, from (1.1) and (3.4) we have

$$
\begin{aligned}
F^{(N)}= & (-1)^{N-1}(N-1) ! \sum_{n=0}^{\infty}\left(\sum_{k=1}^{N} \sum_{m_{3}=0}^{n} \sum_{m_{2}=0}^{m_{3}} \sum_{m_{1}=0}^{m_{2}}\right. \\
& \times(-1)^{n+m_{1}+m_{3}}\left(\begin{array}{c}
n \\
m_{3}
\end{array}\right)\left(N+n-m_{3}-1\right)_{n-m_{3}}(k-1) ! k^{m_{1}} \\
& \left.\times \lambda^{N+n-k-m_{2}} S_{1}\left(m_{2}, m_{1}\right) S_{1}\left(m_{3}, m_{2}\right) H_{n-1, k-1}\right) \frac{t^{n}}{n !} .
\end{aligned}
$$

By equating (3.2) and (3.5), we finally get the following theorem.

Theorem 3.1. For $\mathrm{N}=1,2,3, \cdots$, and $\mathrm{n}=0,1,2, \cdots$, we have

$$
\begin{aligned}
(-1)^{N+n+1} & \frac{(n+N) !}{(N-1) !} \sum_{l=0}^{N+n-1} \frac{D_{l, \lambda}}{l !} \frac{(-\lambda)^{n+N-l-1}}{n+N-l} \\
= & \sum_{k=1}^{N} \sum_{m_{3}=0}^{n} \sum_{m_{2}=0}^{m_{3}} \sum_{m_{1}=0}^{m_{2}}(-1)^{m_{1}+m_{3}}\left(\begin{array}{c}
n \\
m_{3}
\end{array}\right)\left(N+n-m_{3}-1\right)_{n-m_{3}} \\
& \times(k-1) ! k^{m_{1}} \lambda^{N+n-k-m_{2}} S_{1}\left(m_{2}, m_{1}\right) S_{1}\left(m_{3}, m_{2}\right) H_{N-1, k-1}
\end{aligned}
$$

where $\mathrm{H}_{\mathrm{N}, \mathrm{j}}$ 's are as in (1.2), (1.3) and (1.4).

\section{Acknowledgment}

We would like to thank the referees for their valuable suggestions and comments. The first author is appointed as a chair professor at Tianjin Polytechnic University by Tianjin City in China from August 2015 to August 2019. The present research has been conducted by the Research Grant of Kwangwoon University in 2017. 


\section{References}

[1] A. Bayad, T. Kim, Identities for Apostol-type Frobenius-Euler polynomials resulting from the study of a nonlinear operator, Russ. J. Math. Phys., 23 (2016), 164-171. 1

[2] L. Carlitz, A degenerate Staudt-Clausen theorem, Arch. Math. (Basel), 7 (1956), 28-33. 1

[3] L. Carlitz, Degenerate Stirling, Bernoulli and Eulerian numbers, Utilitas Math., 15 (1979), 51-88.

[4] D. V. Dolgy, D. S. Kim, T. Kim, On the Korobov polynomials of the first kind, (Russian) Mat. Sb., 208 (2017), 65-79.

[5] D. V. Dolgy, D. S. Kim, T. Kim, T. Mansour, Degenerate poly-Bernoulli polynomials of the second kind, J. Comput. Anal. Appl., 21 (2016), 954-966. 1

[6] B. S. El-Desouky, A. Mustafa, New results on higher-order Daehee and Bernoulli numbers and polynomials, Adv. Difference Equ., 2016 (2016), 21 pages. 1

[7] T. Kim, Identities involving Frobenius-Euler polynomials arising from non-linear differential equations, J. Number Theory, 132 (2012), 2854-2865. 1

[8] D. S. Kim, T. Kim, Daehee numbers and polynomials, Appl. Math. Sci. (Ruse), 7 (2013), 5969-5976. 1

[9] D. S. Kim, T. Kim, A note on degenerate poly-Bernoulli numbers and polynomials, Adv. Difference Equ., 2015 (2015), 8 pages. 1

[10] D. S. Kim, T. Kim, Identities arising from higher-order Daehee polynomial bases, Open Math., 13 (2015), 196-208. 1

[11] D. S. Kim, T. Kim, Some identities for Bernoulli numbers of the second kind arising from a non-linear differential equation, Bull. Korean Math. Soc., 52 (2015), 2001-2010. 1, 1

[12] T. Kim, D. S. Kim, A note on nonlinear Changhee differential equations, Russ. J. Math. Phys., 23 (2016), 88-92. 1, 1

[13] T. Kim, D. S. Kim, K.-W. Hwang, J.-J. Seo, Some identities of Laguerre polynomials arising from differential equations, Adv. Difference Equ., 2016 (2016), 9 pages. 1

[14] T. Kim, D. S. Kim, H. I. Kwon, D. V. Dolgy, J.-J. Seo, Degenerate falling factorial polynomials, Adv. Stud. Contemp. Math., 26 (2016), 481-499. 1

[15] T. Kim, D. S. Kim, T. Mansour, J.-J. Seo, Linear differential equations for families of polynomials, J. Inequal. Appl., 2016 (2016), 8 pages. 1

[16] N. M. Korobov, On some properties of special polynomials, (Russian) Proceedings of the IV International Conference "Modern Problems of Number Theory and its Applications" (Russian), Tula, (2001), Chebyshevskiū Sb., 1 (2001), 40-49. 1

[17] H. I. Kwon, T. Kim, J.-J. Seo, A note on degenerate Changhee numbers and polynomials, Proc. Jangjeon Math. Soc., 18 (2015), 295-305. 1

[18] J. G. Lee, L.-C. Jang, J.-J. Seo, S.-K. Choi, H. I. Kwon, On Appell-type Changhee polynomials and numbers, Adv. Difference Equ., 2016 (2016), 10 pages. 1

[19] E.-J. Moon, J.-W. Park, S.-H. Rim, A note on the generalized q-Daehee numbers of higher order, Proc. Jangjeon Math. Soc., 17 (2014), 557-565.

[20] H. Ozden, I. N. Cangul, Y. Simsek, Remarks on q-Bernoulli numbers associated with Daehee numbers, Adv. Stud. Contemp. Math. (Kyungshang), 18 (2009), 41-48.

[21] J.-J. Seo, S. H. Rim, T. Kim, S. H. Lee, Sums products of generalized Daehee numbers, Proc. Jangjeon Math. Soc., 17 (2014), 1-9.

[22] Y. Simsek, Apostol type Daehee numbers and polynomials, Adv. Stud. Contemp. Math., 26 (2016), 555-566. 1

[23] A. V. Ustinov, Korobov polynomials and umbral analysis, (Russian) Chebyshevskii Sb., 4 (2003), 137-152. 1

[24] N. L. Wang, H.-L. Li, Some identities on the higher-order Daehee and Changhee numbers, Pure Appl. Math. J., 4 (2015), 33-37. 1 